\title{
EVALUACIÓN DE CUATRO NUEVAS FUNCIONES DISCRIMINANTES DEL HRT II PARA EL DIAGNÓSTICO DEL GLAUCOMA
}

\section{EVALUATION OF FOUR NEW DISCRIMINANT FUNCTIONS FOR HRT II IN GLAUCOMA DIAGNOSIS}

\author{
BORQUE E $E^{1}$, FERRERAS A $^{2}$, POLO V ${ }^{2}$, LARROSA JM ${ }^{2}$, ALIAS E ${ }^{1}$, HONRUBIA FM $^{2}$
}

\section{RESUMEN}

Objetivo: Valorar la capacidad diagnóstica de cuatro fórmulas discriminantes diseñadas para el Heidelberg retina Tomograph (HRT) II a partir de nuestra población.

Material y método: Se incluyeron 450 ojos de 450 pacientes. Fueron clasificados según la presión intraocular, morfología papilar en estereofotografías y los resultados de la perimetría automatizada convencional en 101 ojos normales, 126 hipertensos oculares, 121 sospechosos de glaucoma y 102 ojos con glaucoma. Se calcularon las ANOVAs entre los grupos para todos los parámetros globales y las cuatro fórmulas. Se trazaron las curvas ROC para las cuatro funciones discriminantes diseñadas en nuestro hospital y se compararon las áreas bajo las curvas. Se calcularon los valores de sensibilidad para especificidades fijadas en el $80 \%$ y $90 \%$.

Resultados: Las cuatro fórmulas discriminaron bien entre el grupo control y los sospechosos de glaucoma, y entre controles y glaucomas. Para una especificidad fijada en el 90\%, las sensibilidades oscilaron entre el $54 \%$ y $60 \%$ para diferenciar entre sanos y sospechosos de glaucoma, y entre el $65 \%$ y

\begin{abstract}
Purpose: To assess the diagnostic usefulness of four linear discriminant functions for Heidelberg retina tomograph (HRT) II obtained in our population.

Methods: 450 eyes of 450 patients were studied. Eyes were classified as those of healthy subjects (101), those with ocular hypertension (126), those suspected to have glaucoma (121) or those with glaucoma (103). Intraocular pressure, optic nerve head appearance in stereophotographs, and standard automated perimetry results were assessed. ANOVAs among the groups were calculated for all global parameters and the 4 formulas. Receiver operating characteristic (ROC) curves were plotted for the 4 multivariate functions designed in our hospital and the areas under the ROC curves were compared. Sensitivity at $80 \%$ and $90 \%$ fixed specificities were also calculated.

Results: All functions discriminated well between healthy and glaucoma suspects, and between healthy and glaucomatous eyes. At $90 \%$ fixed specificity, sensitivities ranged from $54 \%$ to $60 \%$ in differentiating between healthy and glaucoma suspects, and from $65 \%$ to $68 \%$ in discriminating bet-
\end{abstract}

\footnotetext{
Recibido: 3/9/07. Aceptado: 13/5/08.

Servicio de Oftalmología. Hospital Universitario Miguel Servet. Instituto Aragonés de Ciencias de la Salud. Zaragoza. España.

1 Licenciado en Medicina.

2 Doctor en Medicina.

Correspondencia:

Enrique Borque Rodríguez-Maimón

Consultas Oftalmológicas. Hospital Universitario Miguel Servet

Isabel la Católica, 1-3

50009 Zaragoza

España

E-mail: eborque@gmail.com
} 
$68 \%$ para diferenciar entre sanos y glaucomas. No se observaron diferencias en las áreas bajo la curva de las distintas fórmulas.

Conclusiones: Las fórmulas discriminantes evaluadas aumentaron la capacidad diagnóstica de los parámetros aislados del HRT II para detectar glaucomas. Asimismo, mostraron mejor rendimiento que las fórmulas discriminantes incluidas en el HRT.

Palabras clave: HRT, diagnóstico, disco óptico, fórmula discriminante.

\section{INTRODUCCIÓN}

El glaucoma primario de ángulo abierto es una neuropatía multifactorial y progresiva caracterizada por la pérdida adquirida de células ganglionares y sus axones en la retina. Junto con la pérdida de fibras nerviosas habitualmente aparecen cambios típicos en la cabeza del nervio óptico y defectos en el campo visual $(1,2)$. La valoración clínica de la cabeza del nervio óptico ha constituido un pilar imprescindible en el diagnóstico y seguimiento del paciente glaucomatoso (3-8). Sin embargo, la evaluación clínica o mediante estereofotografías está sujeta a la experiencia y subjetividad del observador. Estas circunstancias han condicionado que durante los últimos años se hayan desarrollado técnicas no invasivas para estudiar la morfología de la cabeza del nervio óptico de forma rápida, objetiva y reproducible, que supongan una menor dependencia del explorador.

El Heidelberg Retina Tomograph (HRT) es un láser confocal de barrido que ofrece mapas topográficos precisos del disco óptico y de la retina peripapilar. Ofrece una serie de parámetros morfométricos papilares globales y para cada uno de los seis sectores en los que divide la papila, a partir de una reconstrucción tridimensional de la misma. El HRT II dispone de clasificaciones diagnósticas, como la regresión de Moorfields (9), y de herramientas de análisis para el seguimiento.

Diversos estudios $(10,11)$ han buscado mejorar la capacidad diagnóstica del aparato mediante la combinación de los parámetros estereométricos de mayor peso en fórmulas discriminantes. Las herramientas de análisis discriminante lineal combinan diversas variables baremadas en función de su importancia relativa para producir una fórmula de ween control and glaucoma subjects. No differences were found between the areas under the ROC curves of these functions.

Conclusions: The evaluated linear discriminant functions increased the diagnostic ability of HRT II isolated parameters in detecting glaucoma. These functions performed better than the HRT-provided discriminant functions (Arch Soc Esp Oftalmol 2008; 83: 349-356).

Key words: Glaucoma, HRT, diagnosis, optic disc, discriminant function. regresión logística (FRL). El software del HRT incluye dos funciones de este tipo: RB (10) y FSM (11). Bathija (12) y colaboradores han propuesto otra FRL que el software del aparato no incluye pero que ha demostrado poseer una gran capacidad diagnóstica para el glaucoma (13). Otros autores asimismo han propuesto diversas FRL para el HRT cuyo uso está menos extendido que las anteriores (14-16).

La capacidad discriminativa de las herramientas de análisis suele ser menor que la descrita para las poblaciones originales. Las fórmulas son óptimas para la población utilizada para su cálculo, pero no siempre pueden ser generalizadas a otras poblaciones. El objetivo del presente estudio fue evaluar la capacidad diagnóstica de 4 FRL desarrolladas en nuestro hospital (17), mediante análisis multivariante de los parámetros topográficos obtenidos con el HRT II en una muestra poblacional diferente a la utilizada para conseguir las fórmulas.

Diversos factores pueden modificar la validez interna y externa de un estudio de precisión diagnóstica. Debido a ello, se desarrolló la iniciativa STARD (Standards for Reporting of Diagnostic Accuracy) (18). El propósito de la iniciativa STARD es mejorar la calidad de los estudios de habilidad diagnostica. El diseño de este estudio se realizó de acuerdo a los 25 puntos definidos en la iniciativa STARD.

\section{SUJETOS, MATERIAL Y MÉTODOS}

\section{Sujetos}

De enero de 2005 a junio del 2006 se seleccionaron un total de 450 ojos de forma prospectiva y consecutiva a partir de las consultas externas del servi- 
cio de oftalmología del hospital universitario Miguel Servet de Zaragoza.

El diseño del estudio fue aceptado por el comité de ética del hospital, todos los participantes firmaron el consentimiento informado y la metodología del estudio se realizó siguiendo las directrices de la Declaración del Helsinki.

Cada sujeto, con independencia de su grupo de clasificación, cumplió una serie de criterios de inclusión, que fueron: edad entre 30 y 75 años de edad, agudeza visual mayor o igual a 20/30 (escala de Snellen), defectos de refracción menores de 5 dioptrías esféricas y astigmatismo menor de 3 dioptrías de equivalente esférico y medios ópticos transparentes (color u opalescencia nuclear, cortical o subcapsular posterior del cristalino <1 según la Lens Opacities Classification System III) (19).

Se excluyeron aquellos individuos con antecedentes de cirugía o traumatismos oculares graves, enfermedades sistémicas de repercusión oftálmica, imposibilidad de realizar o valorar cualquiera de las pruebas incluidas en el protocolo exploratorio (estudio perimétrico, HRT,...) o por incumplir algún criterio de inclusión.

Se consideró para el estudio un ojo de cada sujeto. La elección se hizo de forma aleatoria, salvo que sólo uno de los dos ojos cumpliera los criterios de inclusión.

\section{Procedimiento exploratorio}

A todos los sujetos se les realizó una exploración oftalmológica completa, que incluyó biomicroscopía con lámpara de hendidura, gonioscopía, medición de los niveles de presión intraocular (PIO) basal mediante tonometría de aplanación (media de 3 tomas en días diferentes sin tratamiento hipotensor), paquimetría corneal central (DGH Technology, modelo DGH 500), evaluación del fondo de ojo mediante oftalmoscopía indirecta y en lámpara de hendidura (con lente Volk de -78 dioptrías), estereofotografías de la papila, perimetría automatizada convencional (PA) y topografía de la cabeza del nervio óptico con el Heidelberg Retina Tomograph (HRT II).

La evaluación clínica del nervio óptico fue realizada con lámpara de hendidura, oftalmoscopía indirecta y estereofotografias papilares por dos especialistas en glaucoma enmascarados con respecto a la historia clínica del sujeto. Las discrepancias entre observadores fueron resueltas por consenso. Se definió la apariencia del nervio óptico compatible con glaucoma como la existencia de un adelgazamiento del anillo neurorretiniano, focal o difuso con aumento de la excavación, la presencia de muescas o ambas (20).

Las PAs se realizaron con el Analizador de Campo «Humphrey 745» (estrategia 24-2 SITA Standard). Los defectos perimétricos fueron definidos por la presencia de un grupo de al menos tres puntos alterados con un nivel de probabilidad menor a $5 \%$ o un grupo de al menos dos puntos alterados con un nivel de probabilidad menor a $1 \%$ (se excluyeron los puntos situados en los polos de la mancha ciega) (21) y/o desviación estándar de la media (DSM) con un nivel de probabilidad menor al 5\%; y/o prueba del hemicampo glaucomatoso fuera de límites normales. Se realizaron al menos dos perimetrías para disminuir el efecto aprendizaje, y si alguna de ellas no cumplió los criterios de validez definidos por el propio perímetro (falsos positivos, falsos negativos y pérdida de fijación), la prueba fue repetida.

El estudio topográfico papilar se realizó empleando un láser confocal de barrido Heidelberg Retina Tomograph versión II que permite obtener una imagen topográfica tridimensional de la papila a partir de tres series de 16 a 64 cortes tomográficos realizados a diferente profundidad y centrados en la cabeza del nervio óptico. La exploración con HRT II fue llevada a cabo de manera estandarizada por un único examinador experimentado. Para su realización se dilataron las pupilas de los pacientes con tropicamida al $1 \%$. Una vez obtenida la imagen digital del disco óptico es necesario delimitar su contorno. El mismo experto en planimetría que desconocía cualquier dato clínico del paciente trazó el contorno de la papila siguiendo el margen interno del anillo escleral de Elschnig. A partir de aquí, el software del HRT II (Heidelberg Eye Explorer versión 1.4.1.0) establece automáticamente un plano de referencia situado 50 micras por debajo de la superficie retiniana del sector temporal comprendido entre 350 y $356^{\circ}$ y calcula una serie de parámetros globales y por sectores de la cabeza del nervio óptico.

\section{Clasificación en grupos}

Los sujetos incluidos fueron clasificados en cuatro grupos de estudio: normal o control, hiperten- 
sión ocular (HTO), sospechosos de glaucoma y glaucoma crónico simple.

- Grupo control (101 ojos): Exploración oftalmológica normal, PIO menor de $20 \mathrm{mmHg}$, morfología del nervio óptico normal y PA sin defectos.

- Grupo de HTO (126 ojos): PIO mayor de 21 $\mathrm{mmHg}$, nervio óptico normal y PA sin defectos.

- Grupo de sospechosos de glaucoma (121 ojos): nervio óptico compatible con glaucoma y PA sin defectos.

- Grupo de glaucoma (102 ojos): PIO mayor de $21 \mathrm{mmHg}$, y PA con defectos glaucomatosos reproducibles.

Con objeto de evitar la posible influencia de los datos morfométricos papilares previos en el análisis de los resultados, los sujetos con glaucoma no fueron seleccionados en base a la evaluación clínica papilar, sino por el resultado de la PA.

\section{Fórmulas discriminantes}

El presente estudio pretende analizar la capacidad diagnóstica de las cuatro FRLs (17) desarrolladas para nuestra población (Zaragoza), en una muestra independiente de la que se utilizó para su diseño. Estas fórmulas son:

FRL 1

$$
\mathrm{P}(\mathrm{Si})=\frac{1}{1+\mathrm{e}^{-(2,348-1,828 \cdot \text { Rim_Area }+4,781 \cdot \text { Cup_Disc }+8,607 \cdot \text { Cup_Shape })}}
$$

Incluye las variables: área de anillo (rim area); relación del área de excavación/disco (cup/disc area ratio) y el índice de morfología de la excavación (cup shape measure).

\section{FRL 2}

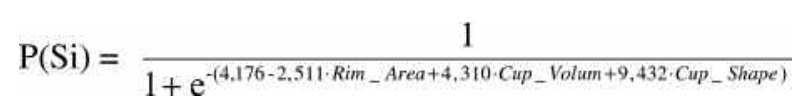

Incluye las variables: area de anillo (rim area), volumen de la excavación (cup volume) e índice de morfología de la excavación (cup shape measure).

FRL 3

$\mathrm{P}(\mathrm{Si})=\frac{1}{1+\mathrm{e}^{-\left(2,157-4,339 \cdot R i m_{-} \text {Volum }+1.358 \cdot \text { Cup _Area }+10,863 \cdot \text { Cup_Shape }\right)}}$

Incluye las variables: volumen de anillo (rim volume), área de excavación (cup area) e índice de morfología de la excavación (cup shape measure).
FRL 4

$\mathrm{P}(\mathrm{Si})=\frac{1}{1+\mathrm{e}^{-\left(3,779-2,534 \cdot \text { Rim }_{-} \text {Area }+1,507 \cdot \text { Cup_Area }+8,404 \cdot \text { Cup_Shape }\right)}}$

Incluye las variables: área de anillo (rim area), área de excavación (cup area), e índice de morfología de la excavación (cup shape measure).

\section{Análisis estadístico}

Para realizar los cálculos y análisis estadísticos se utilizaron los programas estadísticos SPSS 15.0.1. y MedCalc 8.0.1.

Se realizó un análisis de la varianza (ANOVA) de las 4 FRL con el objetivo de valorar la capacidad de discriminar entre los distintos grupos diagnósticos. Se aceptaron diferencias significativas con $\mathrm{p}<0,05$.

Entre los grupos de sujetos normales y sospechosos de glaucoma, y entre los normales y glaucomas se calcularon las curvas ROC (receiver operating characteristic) para cada una de las FRLs. La curva ROC es una representación de la tasa de falsos positivos (1-especificidad) frente a la tasa de verdaderos positivos (sensibilidad). Es una manera útil de mostrar la relación continua entre sensibilidad y especificidad de una prueba o medida. Cuanto más se acerque la curva ROC a la esquina superior izquierda de la gráfica, mejor rendimiento diagnóstico presentará la prueba.

El área bajo la curva (ABC) es un valor numérico que nos da una medida de la capacidad diagnóstica de un test. Una prueba perfecta tendría un ABC de 1 (100\% de sensibilidad y $100 \%$ de especificidad), mientras que un test sin ningún valor diagnóstico tendría un ABC de 0,5. Para establecer si hubo diferencias en las ABCs se utilizó el método descrito por Hanley McNeil (22).

Se calcularon los valores de sensibilidad de las FRLs para especificidades fijadas en el $80 \%$ y $90 \%$, para discriminar entre ojos normales y glaucomatosos.

\section{RESULTADOS}

En la tabla I se describen las características clínicas de los grupos del estudio. No se encontraron diferencias estadísticamente significativas en la edad, la agudeza visual y el valor del espesor corneal central entre los cuatro grupos. La relación excavación/disco vertical valorada mediante estere- 
Tabla I. Características demográficas y clínicas de los grupos del estudio

\begin{tabular}{|c|c|c|c|c|c|c|c|c|c|c|c|c|}
\hline & \multicolumn{3}{|c|}{$\begin{array}{l}\text { Grupo Control } \\
\quad(\mathrm{n}=101)\end{array}$} & \multicolumn{3}{|c|}{$\begin{array}{l}\text { Grupo HTO } \\
(\mathrm{n}=126)\end{array}$} & \multicolumn{3}{|c|}{$\begin{array}{l}\text { Sospechosos de glaucoma } \\
\qquad(\mathrm{n}=121)\end{array}$} & \multicolumn{3}{|c|}{$\begin{array}{l}\text { Grupo de glaucomas } \\
\qquad(\mathrm{n}=102)\end{array}$} \\
\hline & Media & DM & $\mathrm{p}$ & Media & DM & $\mathrm{p}$ & Media & DM & $\mathrm{p}$ & Media & DM & $\mathrm{p}$ \\
\hline Edad & 55,5 & 11,5 & & 53,8 & 12,7 & & 55,7 & 11,2 & & 58,9 & 9,50 & \\
\hline A.V. & 0,89 & 0,188 & & 0,93 & 0,08 & & 0,92 & 0,1 & & 0,85 & 0,12 & \\
\hline PIO basal & 14,6 & 2,79 & $\mathrm{H}, \mathrm{S}, \mathrm{G}$ & 22,9 & 1,55 & $\mathrm{~N}$ & 21,3 & 4,25 & $\mathrm{~N}, \mathrm{G}$ & 24,2 & 4,53 & $\mathrm{~N}, \mathrm{~S}$ \\
\hline $\mathrm{E} / \mathrm{D}$ vertical & 0,27 & 0,22 & $\mathrm{H}, \mathrm{S}, \mathrm{G}$ & 0,43 & 0,27 & $\mathrm{~N}, \mathrm{~S}, \mathrm{G}$ & 0,57 & 0,20 & $\mathrm{~N}, \mathrm{H}, \mathrm{G}$ & 0,71 & 0,18 & $\mathrm{~N}, \mathrm{H}, \mathrm{S}$ \\
\hline Paquimetría & 560,4 & 21,8 & & 563,35 & 37,8 & & 558,5 & 36,1 & & 557,90 & 34,7 & \\
\hline DM de la PA & $-0,56$ & 1,54 & G & $-0,42$ & 1,25 & G & $-0,33$ & 1,29 & $\mathrm{G}$ & $-6,81$ & 6,79 & $\mathrm{~N}, \mathrm{H}, \mathrm{S}$ \\
\hline DSM de la PA & 1,03 & 1,06 & G & 1,11 & 0,75 & G & 1,09 & 1,03 & G & 4,95 & 3,75 & $\mathrm{~N}, \mathrm{H}, \mathrm{S}$ \\
\hline
\end{tabular}

$\mathrm{N}$ : Diferencias con el grupo normal $(\mathrm{p}<0,05)$; H: Diferencias con el grupo de HTO $(\mathrm{p}<0,05)$; P: Diferencias con el grupo de glaucomas pre-perimétricos $(\mathrm{p}<0,05)$; G: Diferencias con el grupo de glaucomas $(\mathrm{p}<0,05)$.

HTO = hipertensos oculares; $\mathrm{DM}=$ desviación media; $\mathrm{AV}=$ mejor agudeza visual corregida; $\mathrm{PIO}$ = presión intraocular; $\mathrm{E} / \mathrm{D}$ = relación excavación/disco vertical en estereofotografías; PA = perimetría automatizada convencional; DSM = desviación estándar de la media.

ofotografías fue diferente en los cuatro grupos, y la PIO basal mostró diferencias entre los normales y el resto de los grupos, y entre los glaucomas pre-perimétricos y los glaucomas. La desviación media $(\mathrm{DM})$ y la DSM presentaron diferencias entre el grupo de glaucomas y los otros tres grupos.

Los ANOVAs de las FRLs mostraron diferencias estadísticamente significativas entre los grupos de HTO y de controles con los grupos de sospechosos

Tabla II. Valores para cada una de las 4 FRLs en los distintos grupos diagnósticos

\begin{tabular}{lccc}
\hline & $\mathrm{N}$ & Media & $\mathrm{p}$ \\
\hline FRL 1 & & & \\
$\quad$ Control & 101 & 0,19 & $\mathrm{H}, \mathrm{S}, \mathrm{G}$ \\
$\quad$ HTO & 126 & 0,36 & $\mathrm{~N}, \mathrm{~S}, \mathrm{G}$ \\
$\quad \begin{array}{l}\text { Sospechosos } \\
\text { Glaucoma }\end{array}$ & 121 & 0,74 & $\mathrm{~N}, \mathrm{H}$ \\
FRL 2 & 102 & 0,76 & $\mathrm{~N}, \mathrm{H}$ \\
$\quad$ Control & 101 & 0,18 & $\mathrm{H}, \mathrm{S}, \mathrm{G}$ \\
$\quad$ HTO & 126 & 0,37 & $\mathrm{~N}, \mathrm{~S}, \mathrm{G}$ \\
$\quad$ Sospechosos & 121 & 0,72 & $\mathrm{~N}, \mathrm{H}$ \\
$\quad$ Glaucoma & 102 & 0,75 & $\mathrm{~N}, \mathrm{H}$ \\
FRL 3 & & & \\
$\quad$ Control & 101 & 0,19 & $\mathrm{~S}, \mathrm{G}$ \\
$\quad$ HTO & 126 & 0,24 & $\mathrm{~S}, \mathrm{G}$ \\
$\quad$ Sospechosos & 121 & 0,70 & $\mathrm{~N}, \mathrm{H}$ \\
$\quad$ Glaucoma & 102 & 0,75 & $\mathrm{~N}, \mathrm{H}$ \\
FRL 4 & & & \\
$\quad$ Control & 101 & 0,21 & $\mathrm{~S}, \mathrm{G}$ \\
$\quad \begin{array}{l}\text { HTO } \\
\text { Sospechosos }\end{array}$ & 126 & 0,36 & $\mathrm{~S}, \mathrm{G}$ \\
$\quad$ Glaucoma & 121 & 0,73 & $\mathrm{~N}, \mathrm{H}$ \\
\hline \hline
\end{tabular}

$\mathrm{N}$ : Diferencias con el grupo normal $(\mathrm{p}<0,05)$; H: Diferencias con el grupo de HTO $(\mathrm{p}<0,05)$; P: Diferencias con el grupo de glaucomas preperimétricos $(\mathrm{p}<0,05)$; G: Diferencias con el grupo de glaucomas $(\mathrm{p}<0,05)$. La columna p representa el resultado de la ANOVA entre los grupos (significación estadística $\mathrm{p}<0,05$ ). de glaucoma y de glaucomatosos en todas las fórmulas (tabla II y fig. 1). Además, las fórmulas FRL 1 y FRL 2 mostraron diferencias estadísticamente significativas entre el grupo control y el grupo de HTO. No se encontraron diferencias entre los sospechosos de glaucoma y los glaucomas para los valores de las cuatro fórmulas, ni entre los controles e HTO para las fórmulas FRL 3 y FRL 4.

A la hora de valorar la capacidad diagnóstica de nuestras fórmulas entre ojos normales y glaucomatosos, el análisis ROC (tabla III, fig. 2) de las FRLs mostró para una especificidad fijada al $90 \%$, unas sensibilidades del 65\% para la FRL 1, del 68\% para la FRL 2 y del $67 \%$ tanto para FRL 3 como para la FRL 4 . De la misma manera, para una especificidad del $80 \%$, las sensibilidades encontradas para las 4 FRLs aumentaron hasta el 76\% y el 77\%. La FRL 4 presentó la mayor $\mathrm{ABC}(0,865)$. No se encontraron diferencias significativas entre las ABCs de las 4 FRLs.

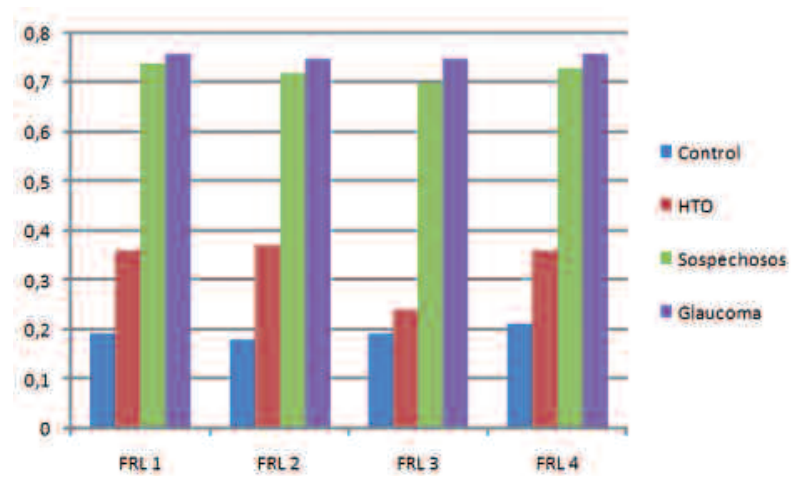

Fig. 1: Valores medios de las cuatro FRLs en los diferentes grupos diagnósticos. 
Tabla III. Áreas bajo la curva ROC para cada una de las FRLs entre sujetos normales y glaucomas

Área bajo la Curva $\quad 95 \%$ IC $\quad \mathrm{p} \quad$ Sensibilidad/especificidad

Especificidad $\geq 90 \% \quad$ Especificidad $\geq 80 \%$

\begin{tabular}{llllll}
\hline FRL 1 & 0,863 & $0,813-0,913$ & $<0,001$ & $65 \%$ & $77 \%$ \\
FRL 2 & 0,863 & $0,813-0,914$ & $<0,001$ & $68 \%$ & $76 \%$ \\
FRL 3 & 0,845 & $0,791-0,900$ & $<0,001$ & $67 \%$ & $76 \%$ \\
FRL 4 & 0,865 & $0,815-0,915$ & $<0,001$ & $67 \%$ & $76 \%$ \\
\hline \hline
\end{tabular}

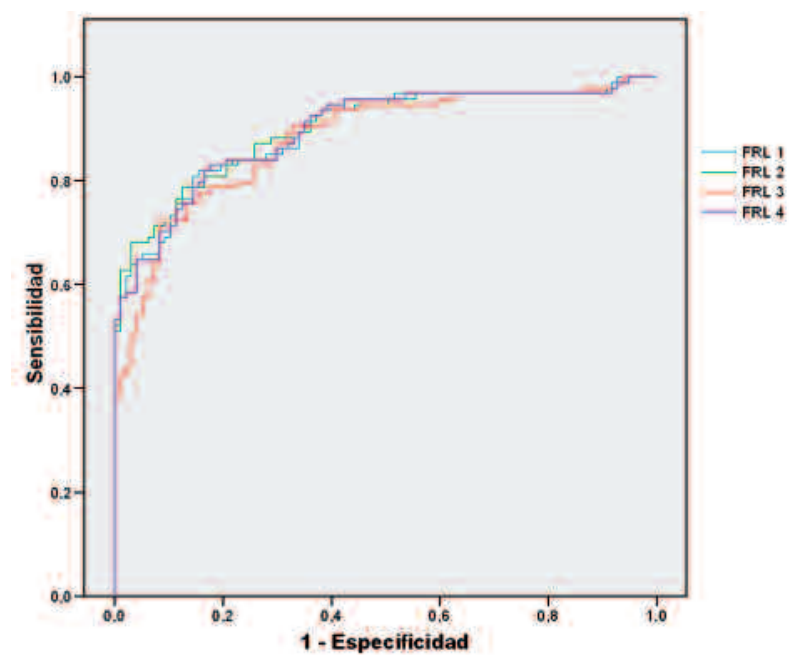

Fig. 2: Curvas ROC para las 4 FRLs entre los grupos control y de glaucomas. No se encontraron diferencias entre las AUCs.

Las ABCs de las FRLs para discriminar entre sujetos normales y sospechosos de glaucoma (tabla IV) fueron discretamente menores que en el caso anterior. Tampoco se encontraron diferencias significativas entre ellas. La mejor ABC se observó para la FRL $1(0,864)$. Para una especificidad fijada en el $90 \%$ las sensibilidades de las 4 FRLs se encontraron entre el $54 \%$ y el $60 \%$. Mientras que para una especificidad fijada en el $80 \%$ las sensibilidades oscilaron entre el $70 \%$ y el $75 \%$.

Tabla IV. Áreas bajo la curva ROC para cada una de las FRLs entre sujetos normales y sospechosos de glaucoma

\begin{tabular}{|c|c|c|c|c|}
\hline \multirow{2}{*}{\multicolumn{2}{|c|}{$\begin{array}{c}\text { Área bajo } \\
\text { la curva }\end{array}$}} & \multirow[t]{2}{*}{$\mathrm{p}$} & \multicolumn{2}{|c|}{ Sensibilidad/especificidad } \\
\hline & & & $\begin{array}{l}\text { Especificidad } \\
\geq 90 \%\end{array}$ & $\begin{array}{c}\text { Especificidad } \\
\geq 80 \%\end{array}$ \\
\hline FRL 1 & 0,864 & $<0,001$ & $60 \%$ & $74 \%$ \\
\hline FRL 2 & 0,854 & $<0,001$ & $54 \%$ & $75 \%$ \\
\hline FRL 3 & 0,847 & $<0,001$ & $55 \%$ & $70 \%$ \\
\hline FRL 4 & 0,863 & $<0,001$ & $59 \%$ & $71 \%$ \\
\hline
\end{tabular}

Entre el grupo control y los glaucomas, las ABCs de las funciones discriminantes incluidas en el software comercializado para el HRT II fueron de 0,850 y 0,798 para la FSM y RB, respectivamente. Para una especificidad fijada al $90 \%$ la sensibilidad fue del 68,6\% para el FSM y del 50\% para el RB. Entre controles y sospechosos de glaucoma, las ABCs fueron de 0,820 para el FSM y 0,689 para el RB. Las sensibilidades fueron de $56,2 \%$ y $23,1 \%$, respectivamente, para especificidades fijadas en el $90 \%$.

\section{DISCUSIÓN}

Para aumentar la rentabilidad diagnóstica de los parámetros morfométricos papilares, el software integrado en el HRT II incluye dos fórmulas discriminantes (RB y FSM). Se ha observado que los valores de sensibilidad y especificidad de dichas fórmulas han sido menores cuando se aplican en poblaciones independientes a aquellas a partir de las cuales fueron diseñadas (23).

Las ABCs obtenidas entre normales y glaucomas para las distintas fórmulas, oscilaron entre 0,845 de la FRL 3 y 0,865 de la FRL 4. Estos valores también fueron algo inferiores a los descritos en la población original, en la que mostraron ABCs entre 0,881 y 0,900, siendo la mejor la de la FRL 2 (17).

Bowd y col. (14) realizaron una comparación entre distintas fórmulas de análisis discriminante publicadas para el HRT II. La mejor fórmula fue una desarrollada en esa misma población (ABC de $0,906 \pm 0,02$ ) pero las $\mathrm{ABCs}$ del resto de las fórmulas estudiadas oscilaron entre 0,848 y 0,890 . Observaron que la que peor rendimiento diagnóstico presentó fue la descrita por Mikelberg y col. (11), con una sensibilidad del $64 \%$ para una especificidad fijada en el $90 \%$.

Mardin (15) propuso una fórmula discriminante que presentó una sensibilidad del $83,6 \%$ para una especificidad del 95\%, para diferenciar entre ojos glaucomatosos y normales. Pero esta capacidad dis- 
minuyó sensiblemente al tratar de diferenciar entre ojos normales y glaucomas pre-perimétricos (sensibilidad del $42,2 \%$ para la misma especificidad del 95\%). En nuestro estudio las 4 FRLs presentaron un mejor rendimiento diagnóstico para diferenciar entre normales y sospechosos de glaucoma.

Se ha visto que el estadio de la enfermedad (medido mediante la puntuación del estudio AGIS) afecta de manera significativa al rendimiento de diversos equipos de imagen utilizados en el diagnóstico de glaucoma, incluido el HRT II (24). La sensibilidad de estos aparatos aumentó al aumentar el nivel de daño por glaucoma en la escala AGIS. Por ello, es difícil comparar los resultados obtenidos en diversos estudios (diferentes criterios de selección de las muestras, y diferente extensión y profundidad de los defectos del campo visual en cada grupo de glaucomas).

La capacidad diagnóstica de las FRLs fue ligeramente mayor que las incluidas en el software del HRT II. En particular, la función discriminante de Burk (RB), presentó una sensibilidad muy baja al intentar discriminar entre sospechosos de glaucoma y ojos sanos.

Ninguna de las cuatro fórmulas analizadas presentó una capacidad diagnóstica suficiente como para permitir usar el HRT como herramienta única para el diagnóstico del glaucoma. Sería necesaria una especificidad alrededor del 95\% (25) para evitar un número excesivamente alto de falsos positivos, especialmente en una enfermedad como el glaucoma que presenta una prevalencia relativamente baja (26). Cuando ajustamos los puntos de corte para satisfacer un criterio así de estricto, poco más de la mitad de los enfermos fue diagnosticada. En la práctica clínica, el diagnóstico del glaucoma se debe basar en la valoración conjunta de todas las herramientas diagnósticas disponibles (perimetría, tonometría, valoración clínica papilar, etc.).

El uso de fórmulas discriminantes mejora la capacidad diagnóstica del topógrafo papilar HRT II, siendo mayor cuando la población objeto del estudio es más parecida al modelo con el que se compara. Las muestras de las que parten las bases normativas incluidas en los distintos sistemas de imagen limitan la capacidad de obtener mayor rentabilidad diagnóstica en poblaciones de características demográficas diferentes. Es recomendable el desarrollo de bases normativas de muestras mayores y más representativas, así como el desarrollo de fórmulas discriminantes multivariantes adaptadas a la población diana. En este sentido la última versión del HRT (HRT3) ha aumentado su base de datos de referencia incluyendo más ojos, con mayor rango en los tamaños de disco y diferentes grupos étnicos. Los sistemas con capacidad de adaptación o aprendizaje como las redes neurales también pueden ayudarnos a mejorar la capacidad diagnóstica del HRT y de otros equipos de diagnóstico por imagen. Por ello serán necesarios estudios que evalúen la aplicabilidad clínica de estos avances.

\section{BIBLIOGRAFÍA}

1. Quigley HA. Neuronal death in glaucoma. Prog Retin Eye Res 1999; 18: 39-57.

2. American Academy of Ophthalmology. Preferred practice pattern: primary open-angle glaucoma. San Francisco: American Academy of Ophthalmology; 2000.

3. Balazsi AG, Drance SM, Schulzer M, Douglas GR. Neuroretinal rim area in suspected glaucoma and early chronic open-angle glaucoma. Correlation with parameters of visual function. Arch Ophthalmol 1984; 102: 1011-1014.

4. Airaksinen PJ, Drance SM, Schulzer M. Neuroretinal rim area in early glaucoma. Am J Ophthalmol 1985; 99: 1-4.

5. Abecia E, Honrubia FM. Retinal nerve fiber layer defects and automated perimetry evaluation in ocular hypertensives. Int Ophthalmol 1992; 16: 239-242.

6. Tuulonen A, Airaksinen PJ. Initial glaucomatous optic disk and retinal nerve fiber layer abnormalities and their progression. Am J Ophthalmol 1991; 111: 485-490.

7. Airaksinen PJ, Tuulonen A, Alanko HI. Rate and pattern of neuroretinal rim area decrease in ocular hypertension and glaucoma. Arch Ophthalmol 1992; 110: 206-210.

8. Jonas JB, Königsrereuther KA. Optic disk appearance in ocular hypertensive eyes. Am J Ophthalmol 1994; 117: 732-740.

9. Wollstein G, Garway-Heath DF, Hitchings RA. Identification of early glaucoma cases with the scanning laser ophthalmoloscope. Ophthalmology 1998; 105: 1557-1563.

10. Burk RO, Noack H, Rohrschneider K, Volcker HE. Prediction of glaucomatous visual fields defects by reference plane independent three-dimensional optic nerve head parameters. In: Wall M, Wild JM. Perimetry Update 1998/99. The Hague: Walter Kugler, 1999; 463-474.

11. Iester M, Mikelberg FS, Drance SM. The effect of optic disc size on diagnostic precision with the Heidelberg retina tomograph. Ophthalmology 1997; 104: 545-548.

12. Bathija R, Zangwill L, Berry CC, Sample PA, Weinreb RN. Detection of early glaucomatous structural damage with confocal scanning laser tomography. J Glaucoma 1998; 7: 121-127.

13. Medeiros FA, Zangwill LM, Bowd C, Weinreb RN. Comparison of the GDX VCC scanning laser polarimeter HRT II confocal scanning laser ophthalmoscope, and stratus OCT optical coherence tomography for the detection of glaucoma. Arch Ophthalmol 2004; 122: 827-837.

14. Bowd C, Chan K, Zangwill LM, Goldbaum MH, Lee TW, Sejnowski TJ, et al. Comparing neural networks and linear discriminant functions for glaucoma detection using con- 
focal scanning ophthalmoscopy of the optic disc. Invest Ophthalmol Vis Sci 2002; 43: 3444-3454.

15. Mardin CY, Horn FK, Jonas JB, Budde WM. Preperimetric glaucoma diagnosis by confocal scanning laser tomography of the optic disc. Br J Ophthalmol 1999; 83: 299-304.

16. Ferreras A, Pablo LE, Larrosa JM, Polo V, Pajarín AB, Honrubia FM. Discriminating between normal and glaucoma-damaged eyes with the Heidelberg Retina Tomograph 3. Ophthalmology 2008; 115: 775-781.

17. Argilés C. Funciones multivariantes de detección de daño glaucomatoso en la cabeza del nervio óptico y determinación de intervalos de normalidad de distribución del anillo neurorretiniano mediante láser confocal HRT-II. Zaragoza: Universidad de Zaragoza. 2005. Thesis doctoralis.

18. Bossuyt PM, Reitsma JB, Bruns DE, Gatsonis CA, Glasziou PP, Irwig LM, et al. The STARD statement for reporting studies for diagnostic accuracy: explanation and elaboration. Clin Chem 2003; 49: 7-18.

19. Chylack LT Jr, Wolfe JK, Singer DM, Leske MC, Bullimore MA, Bailey IL, et al. The Lens Opacities Classification System III. The Longitudinal Study of Cataract Study Group. Arch Ophthalmol 1993; 111: 831-836.
20. Tuulonen A, Airaksinen PJ. Initial glaucomatous optic disk and retinal nerve fiber layer abnormalities and their progression. Am J Ophthalmol 1991; 111: 485-490.

21. Caprioli J. Automated perimetry in glaucoma. Am J Ophthalmol 1991; 111: 235-239.

22. McNeil BJ, Hanley JA. Statistical approaches to the analysis of receiver operating characteristic (ROC) curves. Med Decis Making 1984; 4: 137-150.

23. Ford BA, Artes, PH, McCormick TA, Nicolela MT, LeBlanc RP, Chauhan BC. Comparison of data analysis tools for detection of glaucoma with the Heidelberg Retina Tomograph. Ophthalmology 2003; 110: 1145-1150.

24. Medeiros FA, Zangwill LM, Bowd C, Sample PA, Weinreb $R N$. Influence of disease severity and optic disc size on the diagnostic performance of imaging instruments in glaucoma. Invest Ophthalmol Vis Sci 2006; 47: 1008-1015.

25. Stamper RL. Glaucoma screening. J Glaucoma 1998; 7: 149-150.

26. Wolfs RC, Borger PH, Ramrattan RS, Klaver CC, Hulsman CA, Hofman A, et al. Changing views on open-angle glaucoma: definitions and prevalences-The Rotterdam Study. Invest Ophthalmol Vis Sci 2000; 41: 3309-3321. 